\title{
Anabel Moriña (Ed.) (2021). Enseñando con metodologías inclusivas en la Universidad. De la teoría a la práctica. Madrid: Narcea (Col. Universitaria). 152 págs. ISBN: 978-84-277-2796-0. ePdf: 978-84-277-2797-7. ePub: 978-84-277-2798-4
}

\author{
Alberto José Pazo Labrador ${ }^{1}$ \\ ${ }^{1}$ Universidad de Vigo.apazo@uvigo.es
}

Recibido: $28 / 5 / 2021$

Aceptado: 26/6/2021

\section{Copyright $\mathbb{C}$}

Facultad de CC. de la Educación y Deporte.

Universidad de Vigo

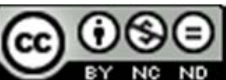

Dirección de contacto:

Alberto José Pazo Labrador

Facultade de Ciencias da Educación e do Deporte

Campus A Xunqueira, s/n

36005 Pontevedra
Enseñando con metodologías inclusivas en la Universidad. De la teoría a la práctica, es el nuevo libro de la profesora Anabel Moriña, en este caso como Editora al frente de un nutrido grupo de profesionales, que se concibe como "un recurso teórico y práctico para revisar y mejorar las prácticas docentes", con una perspectiva inclusiva e integradora, todo un desafío para la sociedad actual y para la educación como un elemento básico de ésta. Es además una fuente de ideas y de recursos muy útil y nutritiva para enriquecer la acción docente, muy necesitada, en estos tiempos líquidos de incertidumbre y sobresaltos casi diarios por la pandemia, de una guía sólida que encauce su inseguro devenir y desarrollo.

Configurado en el marco de un ambicioso proyecto de investigación, el libro tiene como finalidad mostrar ejemplos prácticos de pedagogía inclusiva en diversos centros y contextos universitarios españoles y en diversas áreas de conocimiento, acompañados de los correspondientes sustentos teóricos y metodológicos que los justifican. Para ello se recogen siete estrategias metodológicas activas que se hacen visibles en lo que la editora considera buenas prácticas desde una perspectiva inclusiva: el aprendizaje cooperativo o colaborativo, el aprendizaje-servicio, el aprendizaje por proyectos, las lecciones interactivas, el método de casos, la clase invertida (flipped classrroom) y el aprovechamiento de las tecnologías emergentes. A lo largo de toda la obra se sigue un esquema similar, de manera que al capítulo que sirve de descripción pormenorizada de las bases teóricas, conceptuales y metodológicas de la estrategia, le siguen uno o varios que relatan y fundamentan casos de implementación en diversas universidades y titulaciones españolas, que sirven de ejemplo de buena práctica, como antes indicamos, y también enmarcados en una articulación común: descripción de la estrategia metodológica y fundamentación teórica, ideas para su puesta en práctica (tareas, actividades, estructura, recomendaciones) y concreción de sus beneficios para la mejora del proceso de aprendizaje y para la inclusión de todo el alumnado.

El primer capítulo está a cargo de la propia Anabel Moriña y se titula Estrategias de enseñanza y aprendizaje inclusivas, con una aproximación teórica a sus principios y enfoques. Asumiendo la necesidad -la obligación moral de hecho- de unas 
prácticas docentes inclusivas como una cuestión de derecho y de justicia social, que satisfagan las demandas educativas del mayor número de estudiantes posible, para posibilitar que todos y todas culminen con éxito sus estudios, estas estrategias deben articularse poniendo el foco en el aprendizaje del alumnado, que debe ser activo dentro de un contexto de interacción y cooperación y teniendo en cuenta la diversidad de maneras de aprender. La participación e implicación activa del estudiante favorece la motivación, da sentido a lo aprendido, fomenta la sensación de pertenencia y frena el abandono de los estudios.

La estrategias deben ser diversas y buscar la motivación y la implicación con un papel muy importante de las "interacciones positivas" entre el profesorado y el alumnado, que potencien el componente emocional y afectivo, que es fundamental para una educación verdaderamente inclusiva. Como considera la autora, estas estrategias de enseñanza y aprendizaje que apuestan por la inclusión, mejoran y enriquecen el currículo y mejoran los resultados académicos de todos los estudiantes.

El capítulo 2, a cargo de Ana Domenèch y Arecia Aguirre, justifica las bases teóricas y metodológicas de El rompecabezas o Puzzle de Aronson como estrategia para aprender cooperativamente (hay un error en el índice donde se dice colaborativamente). Teniendo en cuenta que "la cooperación no es una característica innata en el ser humano, sino que debe aprenderse y desarrollarse a lo largo de la vida" (pág. 18), debe incidirse en una serie de principios fundamentales para conseguir su desarrollo: la interdependencia positiva entre los estudiantes, con una asunción de responsabilidades y definición de roles dentro del grupo, y la conciencia de que es imprescindible la aportación de todos y todas para la consecución de los objetivos finales; la interacción cara a cara, que supone una reciprocidad en el aprendizaje y el intercambio de ideas y reflexiones; la responsabilidad individual que redundará en el éxito final del trabajo del grupo; el desarrollo de habilidades interpersonales y sociales; y la reflexión, tanto conjunta como individual. A continuación se describe cómo organizar esta estrategia y cuáles son las etapas o fases que deben articular el puzzle de Aronson. Sustentadas en la literatura, las autoras relatan los beneficios de esta estrategia para el aprendizaje y la inclusión de todo el alumnado. Y estos beneficios se ven en la práctica con la implementación de la estrategia, que relata Juan Pedro Navarro García en el capítulo 3, Puesta en práctica de la técnica Puzzle de Aronson en Ciencias de la Educación, en la Universidad Jaume I de Castellón, en el Grado de Maestro en Educación Primaria, en la materia "Organización Educativa" y trabajando unos contenidos a priori poco atractivos, como son las leyes educativas españolas de las últimas décadas.

El capítulo 4, Aprendizaje-Servicio en contextos universitarios para incluir a todo el alumnado, está a cargo de Noelia Melero Aguilar y Almudena Cotán Fernández. Esta estrategia, en esencia, permite el desarrollo más completo del currículo al integrarlo en una práctica real, que está al servicio de las necesidades de la comunidad, lo que permitirá a los estudiantes reflexionar para construir su propio conocimiento. Los aprendizajes académicos se combinan con la prestación de servicios a la comunidad, por lo que en esta estrategia el espacio de aprendizaje "sale" del aula y se abre a la sociedad, lo que permite una fructífera conexión con la realidad social y profesional, comprometiendo al alumnado con su aprendizaje y desarrollando competencias clave, habilidades y destrezas. Las autoras describen 
igualmente las claves para su implementación e incluyen una reflexión sobre sus beneficios, que son de diversa índole y que estimulan la inclusión del alumnado diverso en el aula, además de aunar el aprendizaje con el compromiso social, y la comprensión de problemas reales que afectan a la sociedad y a los que se puede aportar solución: es así, por ejemplo, en el caso de la aplicación al trabajo con colectivos vulnerables o con personas en riesgo de exclusión, con los que se adquiere una responsabilidad que carga de pleno sentido al currículo. Estas bases se desarrollan en el capítulo 5, a cargo de Carmen Miguel Vicente, AprendizajeServicio. El compromiso del Trabajo Social en la Universidad Complutense de Madrid, y en el 6, por Mayka García García, Aprendizaje y Servicio para aprender y enseñar educación inclusiva en Ciencias de la Educación (de nuevo una disonancia con el índice), el primero de ellos aplicado al Grado de Trabajo Social, y el segundo al Grado en Educación Infantil, en la Universidad de Cádiz, y concretamente para una asignatura muy específica sobre inclusión educativa como es "Cultura, Políticas y Prácticas inclusivas en Educación Infantil”. Del primero nos quedamos con la fórmula que propone la autora para desarrollar este proyecto y que es, sin duda, toda una declaración de intenciones: Universidad Formativa + Universidad de la Vida: Universidad del Éxito; del segundo, con la variada panoplia de servicios que la autora ha desarrollado en sus proyectos docentes a lo largo de los años con su alumnado: rutas solidarias para recaudar fondos para una entidad, campañas de difusión de la labor de asociaciones, elaboración de recursos, juegos y materiales didácticos, actividades de ocio compartido, etc., y con las recomendaciones que ofrece para favorecer la inclusión, dentro de su bien articulada propuesta.

El capítulo 7 está firmado por Inmaculada Orozco y Rosario López Gavira, y trata sobre El Aprendizaje Basado en Proyectos como estrategia para el aprendizaje y la participación activa de todo el alumnado. Se trata de una línea metodológica muy en boga en todos los niveles educativos que comporta, en el ámbito universitario, una serie de beneficios para el aprendizaje, la integración y la participación de todo el alumnado, redundando en el éxito profesional futuro. Fundamentado en la máxima, ya no debatible, de que se aprende (también) haciendo, el alumno se convierte en un agente reflexivo, que aborda un problema relevante de su contexto social y puede contribuir, con su trabajo, a resolverlo y/o transformarlo positivamente. Se trata de poner en práctica, en situaciones reales o replicadas, los conocimientos teóricos para aportar soluciones viables a problemas que despierten el interés del propio alumnado, derivados de acontecimientos o situaciones cotidianas que supongan un aldabonazo para actuar y cambiar ciertas condiciones de una comunidad, donde va a primar el trabajo en equipo pero fruto del esfuerzo individual, y donde cada membro del grupo podrá aportar en función de sus diversas posibilidades y ritmos de aprendizaje, recibiendo el apoyo de los demás. El alumnado es parte activa y responsable del proceso de aprendizaje, y puede observar, en la práctica, la utilidad de lo que aprende. Es protagonista del proceso educativo, con la toma de decisiones en grupo y de forma consensuada, sobre la planificación, la temporalización y las acciones que han de llevarse a cabo en cada momento del proceso de realización del proyecto. Como dicen las autoras, sus beneficios son indudables, al mejorar las relaciones entre estudiantes y docentes, al fomentar el aprendizaje real y el desarrollo de nuevas habilidades, y al fomentar la pertenencia e integración-inclusión a un grupo. El 
capítulo 8, a cargo de $\mathrm{M}^{\mathrm{a}}$ Rocío Bohórquez Gómez-Millán desarrolla el tema Deporte desde todos y para todos. Una experiencia de enseñanza-aprendizaje basada en el Aprendizaje por Proyectos, sobre una experiencia en la Universidad de Sevilla, en el Grado de Ciencias de la Actividad Física y el Deporte y en la materia "Psicología Social del Deporte", centrándose en el caso de la gestión de la creación y desarrollo de equipos deportivos como situación-problema real y significativa para el futuro profesional. El capítulo 9, El Trabajo por Proyectos en los Másteres Universitarios. La resolución de problemas como herramienta didáctica en Filología Inglesa, está a cargo de Juan Carlos Palmer Silveira y se centra en el Máster Universitario en Lengua Inglesa para el Comercio Internacional en la Universidad Jaume I, con el relato pormenorizado de dos experiencias, una en el contexto de la propia Universidad y otra inserta en un proyecto internacional con varias universidades, de forma virtual, que ponen el acento en el dominio de tecnologías y metodologías colaborativas y de comunicación dentro de un ambiente empresarial real, con el resultado constatado de conseguir una buena inserción profesional. Y el capítulo 10, Aprendizaje por Proyectos a través de viajes pedagógicos inclusivos. Embarcando con maletas histórico-educativas, a cargo de Pablo Álvarez Domínguez, de la Universidad de Sevilla, describe una propuesta original llevada a cabo en el Grado de Educación Infantil en la asignatura "Corrientes Contemporáneas de la Educación. Implicaciones en la etapa infantil". Se trata de una estrategia concebida como proyecto, problema y trabajo de campo, que permitirá comprender e interpretar la evolución del hecho educativo a través de un viaje imaginario, que realizan los grupos de estudiantes, a lo largo de la historia de la educación contemporánea. Para ello, y poniendo en juego una alta dosis de creatividad, deberán diseñar y elaborar unos objetos pedagógicos que expliquen los conocimientos adquiridos, depositados en una maleta pedagógica, y reconstruidos a través de una historia que se materializa en una representación teatral. Sin duda es una propuesta novedosa y experimentadora, que según el autor facilita la inclusión al ofrecer una puerta abierta al desarrollo de las capacidades de todo el alumnado.

El capítulo 11 aborda el tema Lecciones interactivas. Una lección magistral que coloca al alumnado como protagonista del proceso de enseñanza-aprendizaje, y de él se ocupan María Nieves Sánchez Díaz y Víctor Manuel Molina. También llamada lección magistral participativa, esta estrategia constituye una adaptación de la tan denostada exposición del docente que se combina con la interactuación del alumnado y el trabajo cooperativo en su desarrollo, una interactuación y participación que está perfectamente medida y diseñada para que alcance su mayor efectividad. Para ello es muy importante preparar lo que los autores denominan "estructura de incentivos", concebidos como tareas o actividades que se propondrán a lo largo de la sesión para afianzar la comprensión de los contenidos y posibilitar la consecución de los objetivos de aprendizaje, fomentando la participación del alumnado a través de la motivación. Es por ello que, desde los "disparadores de compromiso" -algo así como la chispa que enciende la motivación y el interés desde el comienzo de una sesión expositiva- hasta las tareas interactivas, deben estar diseñadas cuidadosamente e insertas en el momento justo para garantizar la atención, la participación y la interacción docente-alumno y alumno-alumno. La materialización de esta estrategia se observa en el ejemplo aportado en el capítulo 12 por Miguel Jesús Bascón Díaz, 
titulado Una estrategia didáctica interactiva para la construcción social y compartida del conocimiento en el aula en el Grado de Psicología, experiencia reportada para la Universidad de Sevilla, en el Grado de Psicología en la asignatura "Psicología del Pensamiento y el Lenguaje", para tratar el papel del momento histórico y cultural en la formación del lenguaje, y concretamente en la configuración de localismos lingüísticos. Con esta estrategia, el docente asegura conseguir crear una atmósfera emocional adecuada para el desempeño óptimo del proceso de enseñanzaaprendizaje, además de garantizar la participación activa de todo el alumnado, independientemente de sus capacidades funcionales. $\mathrm{Y}$ en el capítulo 13, Aprendizaje por descubrimiento guiado y fomento del razonamiento inductivo en grupos reducidos de prácticas en Fisioterapia, a cargo de María Jesús Casuso Holgado, del Grado de Fisioterapia de la Universidad de Sevilla, se va un paso más allá, al centrar esta estrategia metodológica en las clases propiamente prácticas, a través de ejemplos o descubrimientos concretos que el docente propicia de forma guiada, que sirven para que el alumnado pueda establecer relaciones, de forma inductiva, y comprender los principios generales. El proceso, como indica la autora, debe estar muy bien medido en sus tiempos para que sea efectivo, y atestigua los beneficios para la inclusión con un caso particular, un alumno con discapacidad que se beneficia de la integración real en un grupo de trabajo y de la creación del conocimiento de forma colaborativa.

El capítulo 14 versa sobre El método de casos. Una estrategia inclusiva para resolver en cooperación situaciones de la realidad profesional y está a cargo de María Dolores Cortés Vega y de Inmaculada Orozco. Esta metodología permite al alumnado enfrentarse durante su formación con situaciones reales para poder conocer en profundidad las características de las facetas de su futura actividad profesional y elaborar juicios y tomar decisiones bien fundamentadas en la teoría. De nuevo se constata el papel activo del discente que construye su aprendizaje de un modo eminentemente práctico, aprendiendo haciendo. Esta experiencia con casos debe complementarse con el diálogo constante con el docente, lo que permitirá conectar con más eficacia los conceptos teóricos y la práctica profesional y potenciar habilidades como la de comunicación. Es una práctica motivadora que, mediante la simulación de situaciones reales o su inmersión directa en ellas, da pleno sentido a los conocimientos teóricos y consolida el aprendizaje, potenciando la reflexión y el diseño creativo de soluciones, en cooperación activa con los iguales dentro de un grupo de trabajo. Los beneficios cristalizan en el fomento del aprendizaje autónomo, la confianza y la seguridad, el desarrollo de habilidades prácticas y en la posibilidad de "escuchar todas las voces" al integrar en el trabajo conjunto de análisis de casos a todo el alumnado que participará de forma activa, aunque tenga distintas necesidades y estilos de aprendizaje. Las experiencias de buenas prácticas con esta estrategia aparecen reseñadas en el capítulo 15, Casos prácticos en la asignatura de Bioquímica del Grado de Medicina, a cargo de Miguel Cerezo, de la Universidad Jaume I, que aúna la resolución de casos con la representación por juego de rol, una experiencia interesante que además del alto grado de implicación permite, según el autor, la reflexión sobre la relevancia del lenguaje que ha de emplearse en la profesión sanitaria, necesitado de adaptarse no solo a la comunicación con los colegas sino a la comunicación adecuada con el paciente o sus familias. Y también en el capítulo 16 Acercando al alumnado a la realidad de la Psicopatología Clínica a través de casos 
prácticos con material audiovisual, por $\mathrm{M}^{\mathrm{a}}$ del Mar Benítez Hernández, del Grado de Psicología de la Universidad de Sevilla. Se trata de una experiencia en la que el estudiante trabaja en clase con personas de diversas patologías, cuando ello es posible, o más frecuentemente a través de grabaciones en video, en las cuales puede implicarse el propio alumnado. El aprendizaje se centra, por tanto, en la relación teoría-caso, un aprendizaje significativo de una asignatura con un lenguaje propio y minucioso que esta estrategia contribuye a fortalecer. A su vez, el trabajo en equipo posibilita la inclusión del alumnado con discapacidad, que se ve beneficiado de la interacción y la motivación del grupo en el desarrollo de los procesos estructurados.

El capítulo 17, Flipped Clasroom o Aula Invertida. Cómo dar la vuelta a la clase, a cargo de Ruth Cabeza Ruiz y Rafael Carballo Delgado, revisa una de las metodologías más de moda últimamente, facilitada por el auge y el perfeccionamiento de las plataformas virtuales de enseñanza y muy adecuada en tiempos de pandemia y desarrollo de la enseñanza no presencial o "no física" como los que vivimos. El papel activo del alumnado en la construcción de su aprendizaje es aquí más evidente ya que es él o ella, fuera de las horas de clase, quien trabaja con el material que, previamente, le ha proporcionado el docente, y es él o ella quien, ya en el aula, planteará dudas, realizará actividades de aplicación práctica, o interactuará con su grupo de iguales para organizar y clarificar los contenidos consolidando el aprendizaje. A la articulación clásica del proceso de enseñanza-aprendizaje se "le da la vuelta" y se consiguen unos resultados muy eficaces, al combinarse en el aula con otras estrategias que fomenten el trabajo en grupo o cooperativo, los proyectos, el estudio de casos, etc. Es un procedimiento muy útil para la inclusión educativa, ya que el material que se proporciona puede ser utilizado y trabajado por cada estudiante a su propio ritmo, en su propio espacio y consumiendo su propio tiempo, no reglado, un material que puede ofrecerse de forma adaptada y flexible. Lógicamente es una estrategia que requiere de una cuidada preparación y planificación, de una coherencia entre la información proporcionada y el desarrollo de la clase presencial donde serán las dudas, las reflexiones, el debate y la interacción del alumnado los que contribuyan a consolidar este modelo de aprendizaje autónomo por antonomasia. La experiencia práctica que se describe en el capítulo 18, Uso del aula invertida en estudiantes universitarios de Ciencias del Deporte, a cargo de Antonio Jesús Sánchez Oliver, así lo atestigua, destacando la importancia de una plataforma o entorno virtual idóneos, así como el uso generalizado de las nuevas tecnologías y canales de comunicación de la vida cotidiana.

Abundando en esto, el capítulo 19 se centra en las Prácticas docentes inclusivas con tecnologías emergentes, y está a cargo de Víctor Hugo Perera Rodríguez y Beatriz Morgado Camacho. Se refieren a tecnologías disruptivas que producen un cambio con respecto a prácticas educativas tradicionales y que suponen profundos cambios en las formas de enseñar y de aprender. Las aplicaciones basadas en tecnología móvil, trasunto de lo que está ocurriendo en todos los ámbitos de la sociedad, tienen cada vez más proyección entre los docentes en busca de fomentar un aprendizaje activo e interactivo, así como más personalizado. Destacan esencialmente la gamificación y la realidad virtual, que es a lo que dedican el capítulo y las dos experiencias posteriores, como herramientas de transformación educativa. La gamificación -que no es tan innovadora; a quien esto escribe le suena incluso a 
rancio: en los años sesenta del siglo pasado, por ejemplo, se introdujeron los juegos en el aprendizaje de algunas Ciencias Sociales como la Geografía; lo que es innovador, en su caso, es su inserción en un entorno digital- favorece la interacción, la participación y la motivación, al crear un entorno de aprendizaje activo y atractivo, rupturista ante las dinámicas tradicionales en el aula, aunque propicia "vicios" como la competitividad (en el fondo otro tipo de "competencia") o la frustración ante la no consecución de "premios" o "emblemas". La realidad virtual, por su parte, permite replicar experiencias del mundo real en ambientes seguros y controlados, abaratando y simplificando determinadas prácticas y experiencias y haciéndolas accesibles a todo el alumnado, facilitando el acceso a la información y la construcción de los aprendizajes. En el capítulo 20, “CConoces tu entorno?”. Un juego de pistas para el aprendizaje de las Ciencias Sociales, por Elisa Navarro Medina, de la Universidad de Sevilla, se describe una estrategia que combina la gamificación con el trabajo de campo investigador y el trabajo por proyectos para conocer el entorno desde el punto de vista de la ubicación espacial y las transformaciones a lo largo del tiempo histórico en los futuros maestros de Primaria. Por último, en el capítulo 21, La realidad virtual en la enseñanza artística universitaria. El espacio $R V$ como modelo pedagógico, está escrito por Yolanda Spinola Elías, de la Universidad de Sevilla, y se centra en el "Máster de Arte, Idea y Producción", y concretamente en los entornos expositivos virtuales y en la producción creativa de obras artísticas virtuales, comprendiendo sus múltiples posibilidades artísticas, estéticas y creativas.

En esencia, se trata de una amplia y representativa panoplia de metodologías innovadoras que animan al docente universitario a explorar nuevos caminos y definir nuevos entornos de aprendizaje, propiciando, en su caso, la inclusión educativa. Me gustaría concluir con una serie de reflexiones que me han surgido a raíz de la lectura del texto:

- Debido al gran número autores implicados, hay una cierta irregularidad en el desarrollo de los capítulos que convive paradójicamente con muchas reiteraciones, a pesar de que debe reconocerse el considerable esfuerzo de la editora por la homogeneización de la estructura de los capítulos. Pero se echa en falta una mayor labor de corrección de estilo y de revisión ortográfica, lo que empaña en ocasiones los contenidos de algunos capítulos. A modo de ejemplo, en varias ocasiones se confunde modelo con estrategia y tecnología con metodología. Habría sido conveniente y clarificador, igualmente, indicar la filiación y el rango académico de cada uno de los autores que se ocupan de cada capítulo.

- La inclusión educativa, que es lo que vende el título del libro, en la práctica no constituye el núcleo del mismo, ya que en no pocas ocasiones aparece tratada de forma colateral o incluso introducida de manera forzada, siendo así que la sensación que queda al terminar de leer el libro es que su temática esencial es reseñar y justificar toda una serie de nuevas estrategias de enseñanzaaprendizaje en la Educación Superior.

- Y a modo de reflexión/provocación, una cuestión emitida por alguien que no duda de las bondades de estas estrategias, y que de hecho utiliza buena parte de ellas desde hace años con muy buenos resultados. ¿Por qué ese afán constante de enfatizar la necesidad de la motivación del alumnado universitario?, cuando: 
1) se trata de personas adultas que han elegido libremente una carrera, por la que muchas veces pagan una pequeña fortuna (no digamos en los centros privados), con lo que la motivación viene "por defecto"; 2) el alumnado sabe a qué va a enfrentarse al iniciar una carrera universitaria, pues como personas adultas responsables tienen el deber, el derecho y la capacidad de conocer un currículum que ha sido elaborado por profesionales competentes tras un largo período de reflexión, por lo que el concepto de "asignaturas aburridas" parece un oxímoron - pintoresco, sino fuera por la trascendencia que tiene asumir tal construcción conceptual-, una contradicción en sus términos y que pone en cuestión la idoneidad de aquellos; 3) al estar facilitando tanto la labor de aprendizaje, y por lo tanto, anatematizando en la práctica el valor del esfuerzo y el trabajo riguroso individual, de la responsabilidad -que se diluye en la colectiva-, de la disciplina, en pos de lo atractivo, lo motivador y lo “divertido”, ¿no estamos “infantilizando” la Universidad? (Frank Furedi dixit). 